\title{
التركيب الفضائي للبنية الحضرية والسلوك الفضائي \\ حالة دراسية شارع المنصور الرئيسي
}

أ.م.د. عامر شاكر الكناني

الباحثة تماره عبد الرزاق محمد

المقدمة:

يعاني شارع المنصور الرئيسي في الوقت الحالي من تغيرات وتحولات غير منظمة للبنية الحضرية اذ ان في الآونة الاخيرة حصلت عدة تغيرات لهذا الثارع من (توسع حضري، ازدياد ارتفاع الابنية، اقحام مراكز تجارية

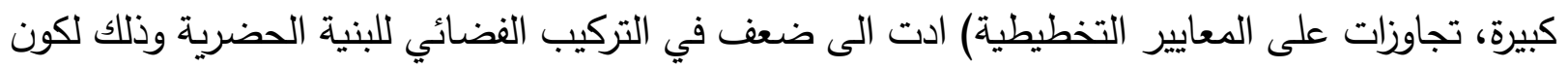
هذه التغيرات لا تعتمد على اسس علمية واضحة مما ادى تغير السلوك الفضائي خلال حركة المتلقي بين مكونات البنية الحضرية من شوارع ومباني. مشكلة البحث: لا يوجد تصور يوضح العلاقة بين التركيب الفضائي للبنية الحضرية مع السلوك الفضائي للمتلقي.

فرضية البحث: السلوك الفضائي للمتلقي يتأثر بتغير الخصائص التركيبية الفضائية للبنية الحضرية.

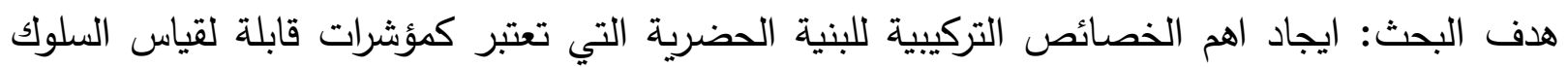
الفضائي للمتلقي. 1 - مفهوم البنية الحضرية:

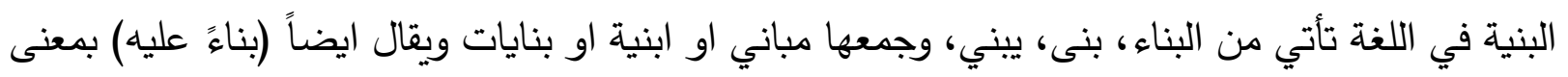

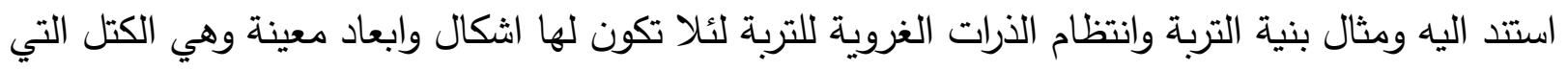

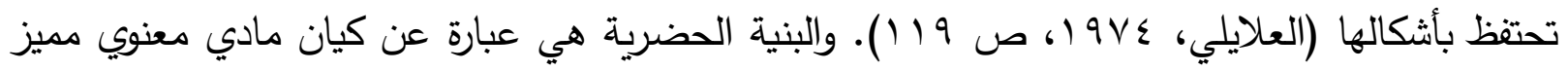
بخصائصه الثكلية، وتتجسد فيها معاني مختلفة تعكس فهم الانسان للبيئة الطبيعية والاجتماعية داخل النسيج 


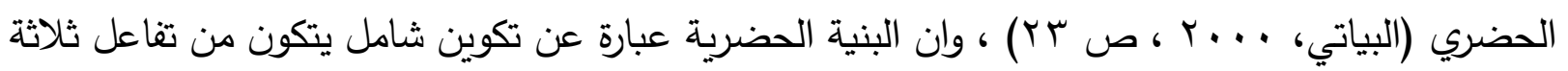
بنى اساسية وهي : (Canter, I9VV, p 101) 1- بنية فضائية (Spatial structure) : تعتبر الحدود المادية المكونة للمحيط البيئي ץ- بنية الفعاليات (السلوكية) (Activity structure) : الاحداث التي ترتبط بمكان معين والسلوكيات التي من المتوقع ان تحدث فيه r- البنية المفاهيمية (Conceptual structure) : وتعني القيم والمفاهيم التي يحملوها الاشخاص الذين يستعملون المكان عن المكان نفسه. وتتفاعل هذه البنيات الثلاثة مع بعضها البعض من خلال متغير مشترك وهو المجتمع والانسان بقدرته الادراكية والاستيعابية.

حلل (Alexander) (مهندس ومنظر معماري) البنية الحضرية لعديد من المدن وعليه توصل الى ان البنية الحضرية للمدن الحديثة طريقة نمو هذه المدن تكون مصممة وتعتمد على ثقافة المصمم الذاتية، سيطرة

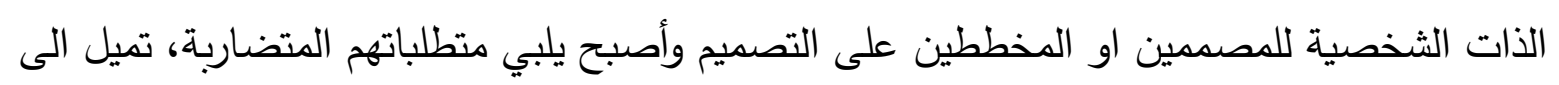
العزل الاجتماعي وفقدان الخصوصية، تمتلك بنية حضرية ذات تكاملات موضعية أكثر مما تكن شمولية .(Alexander, 19^v, p $\leqslant-0 r)$ r- ب العلاقات البنيوية الحسية: هي العلاقات المتعلقة بالجانب المعنوي (الحسي) للمتلقي أكثر من الجانب الثكلي، وتعني هذه العلاقة علاقة الجزء بالكل كما يتحسسها المتلقي إذا يمثل الفضاء الجانب اللامادي اي غير مرئي وملموس هو الكل الكل اما

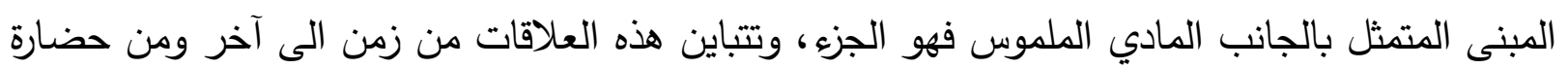
لأخرى. 
r-r r-r التحول في البنية الحضرية: التحول في اللغة يعني تغير او تغيير بالثكل اي تحول الثكل من حال الى اخر من خصائص البنية هي التحولات حيث تم مناقشة التحولات التي تحدث للبنية من خلال عوامل داخلية وهذا يوضي دئ ديناميكية البنية الذاتية، والتي تحدث عن طريق سلسلة من التغيرات داخل المنظومة التي تكون محكومة بقانون البنية الداخلي

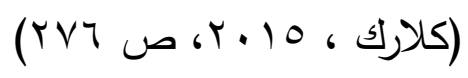

ان التحولات التي تحدث في البنية الحضرية تأخذ جانبين وهما: 1- الجانب الاول هو ان يكون تحول ذاتي والذي يحدث داخل منظومة البنية الحضرية محكوما بقوانين البنية نفسها حيث تعمل هذه القوانين على استيعاب هذا التحول ولا تظهر اي تغيرات شكلية جوهرية وبذلك دون فانه يعتبر تحول يصيب الثكل (المظهر الخارجي) ، ويتميز هذا التحول بالمحافظة على تماسك البنية الحضريـة

r- الجانب الثاني هو التحول القسري ويعمل هذا التحول على تغيير في شكل البنية الحضرية من خلا اقحام عناصر جديدة او تغيير في العلاقات البنيوية لتحقيق اغراض اجتماعية او اقتصادية او سياسية وهذا التحول في الغالب يصيب الجوهر وفي بعض الاحيان يصل هذا التحول الى مرحلة الانقطاع.

r- التركيب الفضائي (Space Syntax):

في اللغة تعني كلمة تركيب مجموعة من القواعد والمبادئ التي تتحكم في علاقة الكلمات مع بعضها لتتشكل العبارة ، فالبنية التركيبية تنتظم بالقواعد والمبادئ، اما في التصميم والتخطيط الحضري تطلق كلمة (Syntax) وتعني العلاقات ما بين الفضاءات او العلاقة بين الفضاء والمجتمع وهذا يؤكد فكرة ان البنية المكانية تؤثر على فعاليات الانسان، وان تركيب الفضاء يقترح لغة برمجة حاسوبية وصفيه لأنماط المدينة المكانية (Hillier, $19 \wedge \varepsilon, p) 7$. 
اذ ان الفضاء يعتبر الوعاء الثامل الحاوي للكتل العمرانية وما بينها من فضاءات (شوارع او ساحات وغيرها) والتي تعتبر المجال الذي يتم به الحركة والنثاطات التفاعلية المجتمعية وهذا يمكن ان نمثله على اساس نظام فراغي الذي يتكون من مجموعة من الفضاءات المستقرة معلوماتيا نسبة لإدراك المستخدمين.

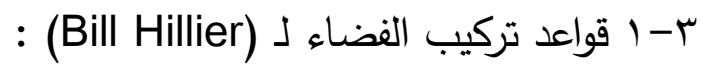
عرف (Hillier) نظرية تركيب الفضاء على انها علاقة بين تولد اشكال البنية الحضرية مع القوى الاجتماعية، وضمت دراسة قواعد تركيب الفضاء ل (Hillier) مجموعة من البحوث التي اجريت في وحدة (Bartlett) للدراسات المعمارية من قبله وعدد من الباحثين حيث تضمنت هذه الدراسات ايجاد العلاقة بين المتغيرات

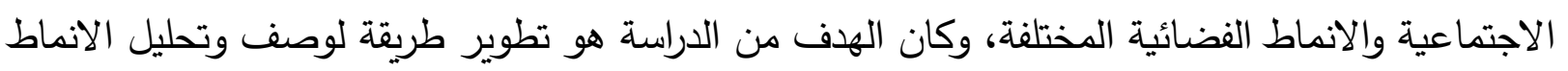

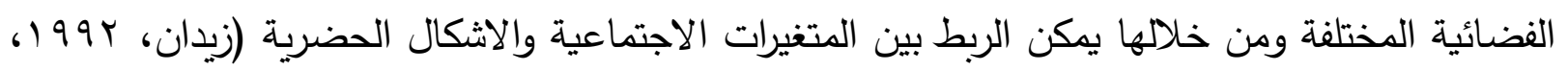

لقد تم تحليل الفضاء وفق هذه القواعد حيث تم اعتبار ان من الضروري ان نفهم الفضاء من منظور وظيفي من حيث ما يفعل الجانب الجوهري للناس فيه اذ ان هناك ثلاث انشطة يقوم بها الناس في الفضاءات هي: التحرك عبر الفضاء، التفاعل الاجتماعي مع الاشخاص الموجودين في الفضاء ، رؤية الفضاء المحيط

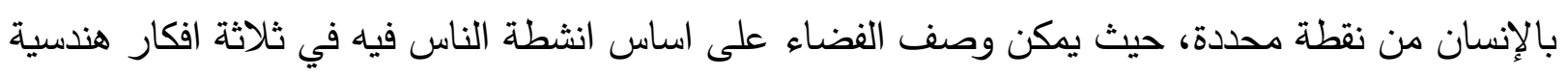
وهي : فضاء خطي (محوري) عندما يتحرك الناس فيه ويتكون من محاور مستقيمة تستوعب حركة الناس ، فضاء تحدبي (حيث يمكن كل نقطة ان ترى النقاط الاخرى) عندما يتفاعلون داخل الفضاء، واخيرا فضاء المجال البصري الذي يرى الفضاء من اي مكان شكلا متغيرا حسب مجال رؤية العين، شكل (1) 


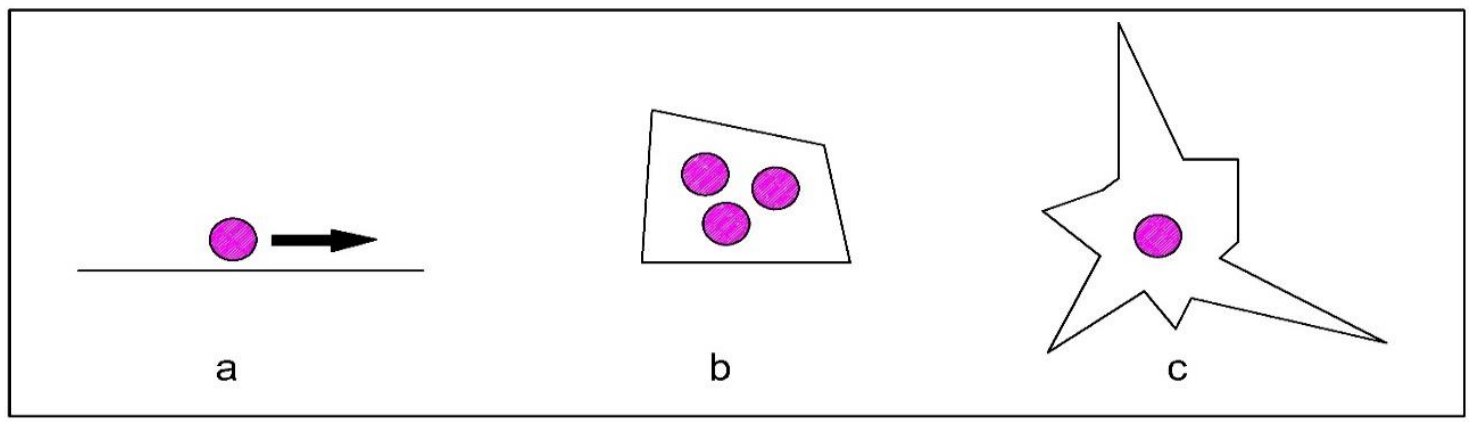

$$
\begin{aligned}
& \text { شكل رقم ( ) وصف الفضاء تركيبيا وفق انشطة الناس / ( a ) فضاء خطي (محوري) (b ) فضاء }
\end{aligned}
$$

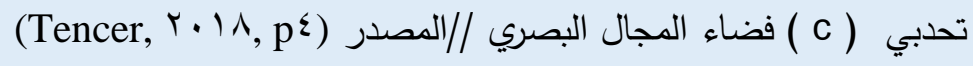

وضح (Hillier) ان الفضاء هو المكان الذي يحدث فيه جميع الانشطة البشرية حيث انه يعتبر انعكاس للفعاليات الاجتماعية والثقافية التي تحدث في المدينة، حيث يتطلب فهم تأثير الفضاء على الناس وكيف لهن يستخدمنه ويتفاعلون فيه مع بعضهم البعض، حيث يذكر (Hillier) عند تمثيل منظومة الفضاء يمكن

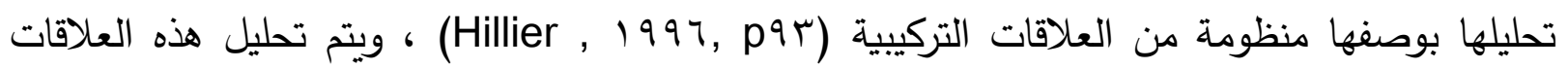
التركيبية وفق خاصيتين اساسيتين: اولا : التناظر واللاتتاظر (Symmetry - asymmetry) : (تون تعبر هذه الخاصية عن الخصائص التنظيمية للفضاءات حيث تعبر عن العمق في العلاقة من حيث العمق

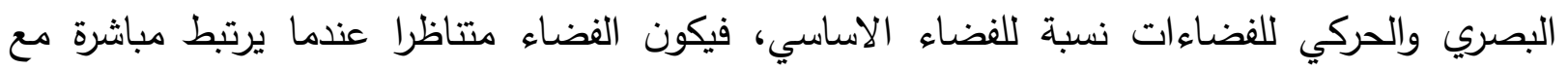
الفضاءات الاخرى كافة، اي انه اقل عمقا نسبة الى كافة الفضاءات الاخرى، ويكون غير متتاظر عندما

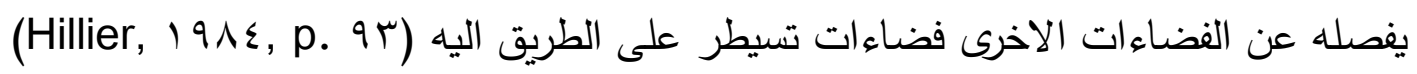
ثانيا : الانتشار واللاانتشار (Distributed - non distributed) : ايضا تعتبر هذه الخاصية من الخصائص التنظيمية للفضاءات فتعبر عن الخيارات في الطرق المتوفرة في النظام للوصول الى كافة الفضاءات، حيث ان الفضاء يعتبر منتشرا في علاقاته عندما يوجد أكثر من طريق 
للوصول اليه واعتبر هذا الفضاء هو فضاء عام، ويكون غير منتشر عندما يوجد طريق واحد فقط يصل اليه واعتبر هذا الفضاء هو فضاء خاص، ولا ترتبط خاصية الانتشار بخاصية التتاظر فالفضاء مدكن ان يكون

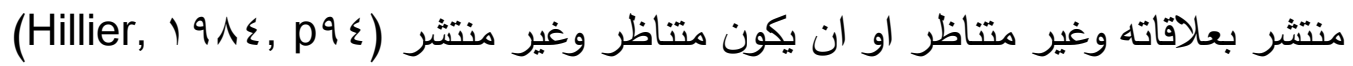

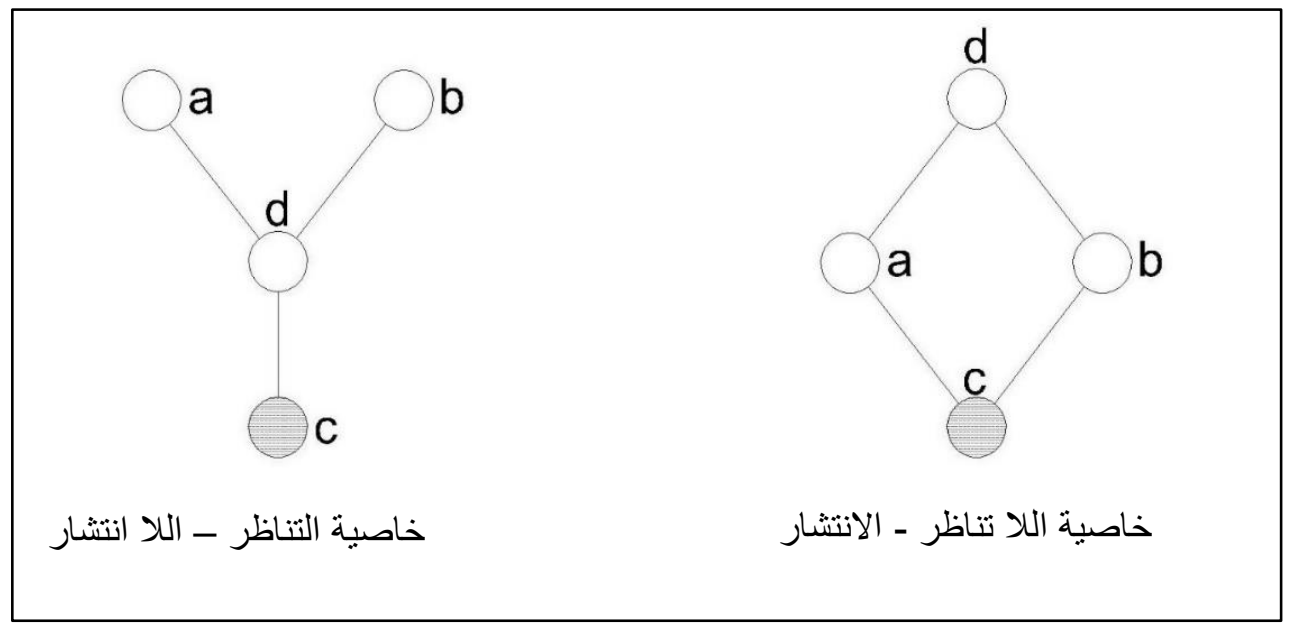

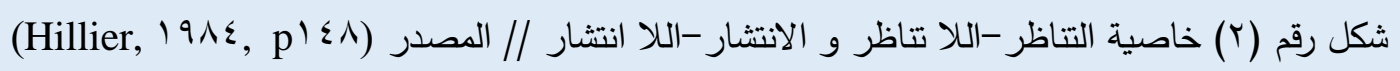

تعد هاتان الخاصيتين هما الخاصيتين الرئيسيتين التي تتميز بها قواعد التركيب الفضائي للبنية الحضرية وتعتبر هي الاساس لباقي الخصائص الاخرى هين r-r الخصائص التركيبية للبنية الحضرية: وضح (Hillier) ان البنية الحضرية عبارة عن تنظيم ثنائي الطرف بين الكتلة البنائية والمحيط الخارجي لها والذي أطلق عليه ايضا (العالم الخارجي) ويمكن ان يتمثل بحدود، وبين ان البنية الفضائية بين هذين الطرفين

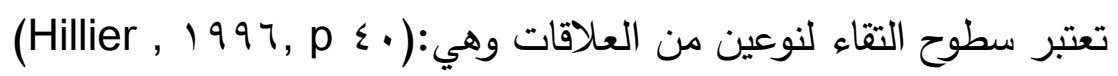
ا- العلاقة الاولى: بين الساكنين، وأطلق عليها خصائص النظام الموضعية، التي تعمل على توجيه حركة الساكن داخل البنية الحضرية التي تحتوي على تفاعلاتهم الاجتماعية. 
r- العلاقة الثانية: بين ساكني النظام والغرباء عنه، وأطلق عليها خصائص النظام الثمولية، مبين ان هذه الخصائص تعمل على تنظيم حركة الغرباء وتقاطعهم مع حركة الساكنين والتي تعتمد على توجيه حركة الغرباء داخل البنية الحضرية. اولا: الخصائص الشمولية: 1- خاصية معدل العمق النسبي (Mean - depth) يعتبر معدل عمق الفضاء في النظام مؤشر لقياس عدم تتاظره النسبي، حيث يهدف معدل العمق الى تحديد

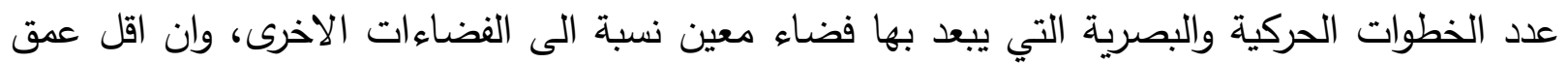

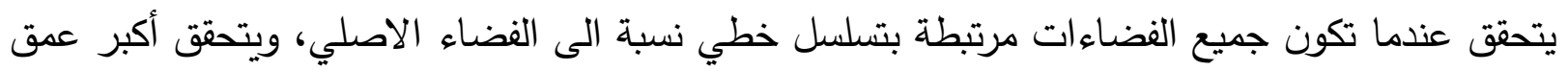

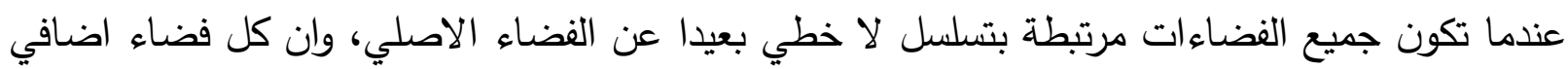
يضيف مستوى اخر للعمق. (1) • Hillier, 1997, p) r- خاصية درجة التكامل الثمولية او خاصية التناظر النسبي: تثير درجة التكامل الثمولية التي يرمز لها (Rn) الى امكانية الاتصال او الوصول لكل الفضاءات ضمن

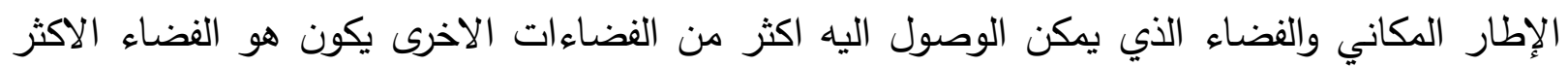
تكاملا، ويكون الافضل للتجمعات وللتفاعل الاجتماعي.

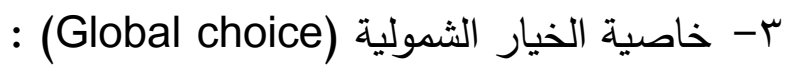
درجة الخيار تثير الى درجة السيطرة التي يحققها الفضاء على نفاذية الفضاءات المجاورة له، وتعتبر

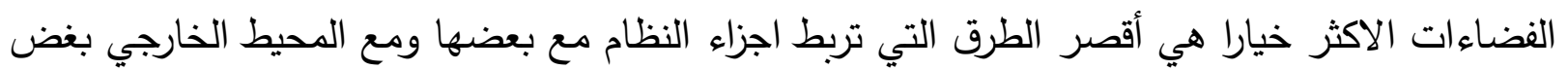

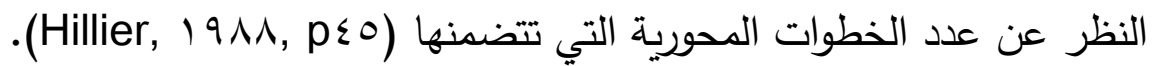
ثانيا: الخصائص الموضعية: هذه المقاييس تحدد طبيعة الخصائص الموضعية اي طبيعة الفضاء الواحد مع الفضاءات المجاورة له بشكل مباشر، وتثمل هذه الخصائص ما يلي: 
(- خاصية الاتصالية (Connectivity)

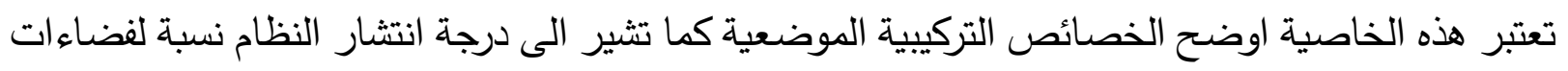
النظام الاخرى، وتحسب قيم الاتصالية للفضاء من مجموع الفضاءات المتصلة به بصورة مباشرة، اي التي

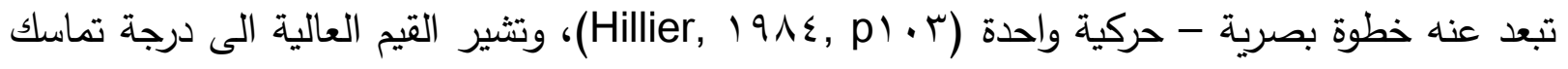

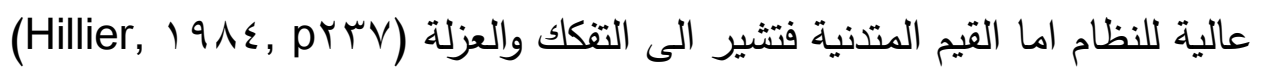
r- خاصية التكامل الموضعي (Local Integration): ويشير الى امكانية الوصول او الاتصال لفضاء معين ضمن جزء من النظام المكاني، وتحتسب هذه القيمة

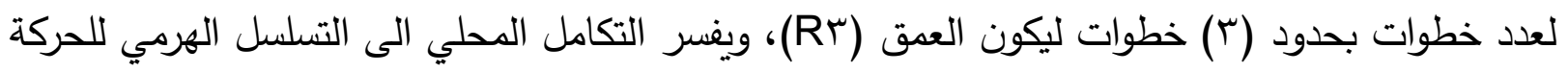
المحلية للمشاة وامكانية وصولهم للفضاء ب- السلوك الحركي (Agent): تون تعد هذه الخاصية من الخصائص المهمة في تحليل حركة المشاة في الفضاء وتوقع الازدحام الحاصل نتيجة للتجمع الحركي في المكان، تحلل هذه الخاصية سلوك الفرد المتنقل في المكان وماهي وجهته وهذا يعتمد ونداء

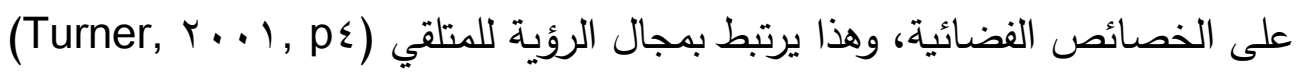
ثالثا: الوضوحية التركيبية للبنية الحضرية (Intelligibility) بين (Hillier) ان البنية الحضرية التي تتميز بالوضوحية الثمولية تمتلك تنوع عالي في اجزاءها الموضعية

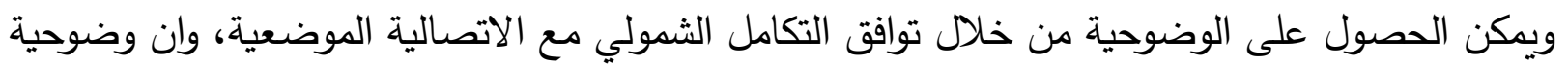
البنية الحضرية تعتمد على حركة المتلقي بين اجزاءها، وبذلك يتم التميز بين الفضاءات التي يرتادها الساكن التصن من الفضاءات التي يرتادها الغريب حيث ان الساكن يتحرك وفق مخططاته الذهنية بين اجزاء المدينة الموضعية التي تتميز بها بيئتهم، اما الغريب فيحاول ان يتحرك في اجزاء البنية الحضرية الواضحة ذات

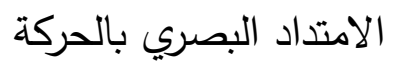




$$
\text { ع - (الادراك: }
$$

يشمل كل العمليات الفكرية من معرفة او فهم تضاف للناتج المعرفي، وهو يعتمد على خزن وتتظيم المعلومات المكتسبة من البيئة المدركة والتي تعرضت للتنكر او التعلم او التفكير او اي تطوير ذهني ولا يهتم بالقضايا

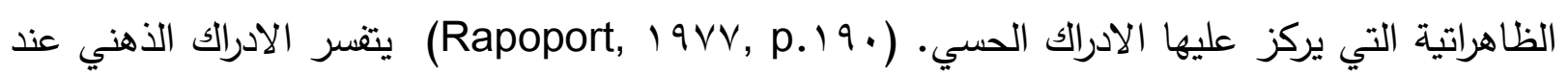
المتلقي على شكل سلوك فضائي (Spatial Behavior) يتخذه في الفضاء ويمكن وصفه، او عن طريق

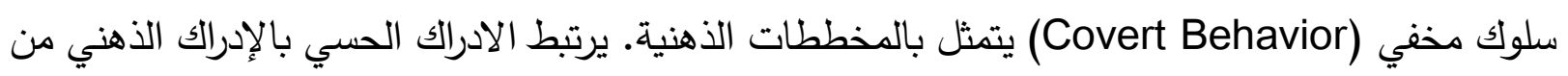
حيث المعلومات الحسية الموجودة في الذاكرة، فهذه المعلومات قد مرت بعثلية بالية الادراك الحسي او كانت عبارة

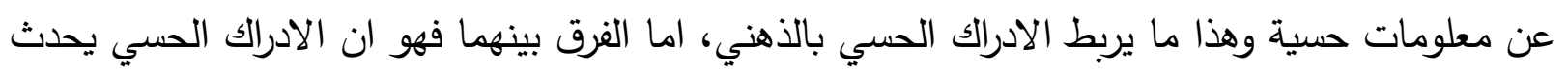

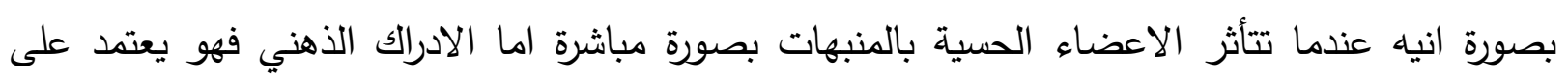

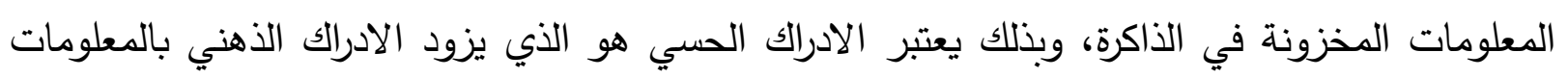

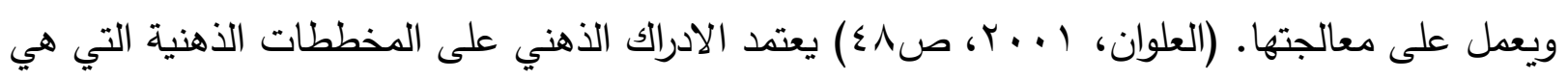
رمز الثكل المخزون في الذاكرة، اي ان ما يخزن هو رمز فقط وليس الثكل ككل، يتم الانتباه الى الاشكال التي لها نفس المخططات وكذلك الاثكال المشابهة لمحاولة اكتثاف عناصر التشابه فيها، وتعتبر تمثيلات داخلية في الذهن لخصائص الاثياء الموجودة في البيئة المحيطة للمتلقي وكذلك موقعها اي انها تعتبر انطباع الناس لبيئته ع- استخلاص مؤشرات البحث

\begin{tabular}{|c|c|c|}
\hline نوع القياس & المفاهيم & المؤشرات \\
\hline وصفي + كمي وفق البرنامج & البنية الفضائية & البنية الفضائية والمفاهيمية \\
\hline وصفي & البنية المفاهيمية & والسلوكية للشارع التجاري ذات | \\
\hline وصفي & البنية السلوكية & خصائص تركيبية تحفز الادراك \\
\hline وصفي + كمي وفق البرنامج & السلوك الفضائي & الذهني وتحدد السلوك الفضائي \\
\hline
\end{tabular}


0- منطقة الدراسة : شارع المنصور الرئيسي: يعد شارع المنصور الرئيسي من اهم شوارع بغداد التجارية أنثأ في ستينات القرن الماضي ويقع في مدينة

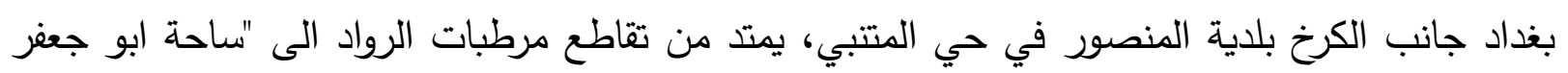

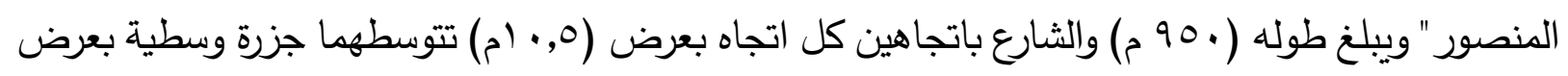

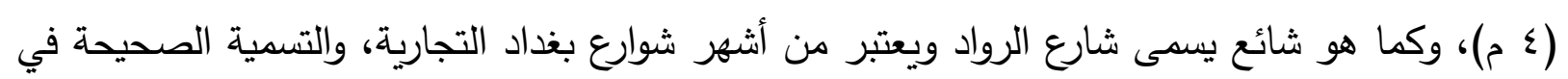

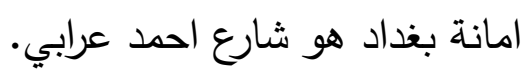
- - تحليل الشارع وفق المؤشرات: البنية الفضائية: يتم تحليل البنية الفضائية بشكل وصفي ووفق برنامج ال • Depthmap تتألف البنية

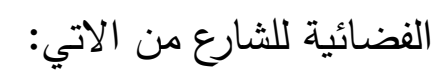
ا - المراكز التجارية: توجد العديد من المراكز التجارية في الثارع وهي: أ- اسواق المنصور المركزية سابقا- مول خليفة حاليا ب- مول المنصور ت- مول التاون سنتر

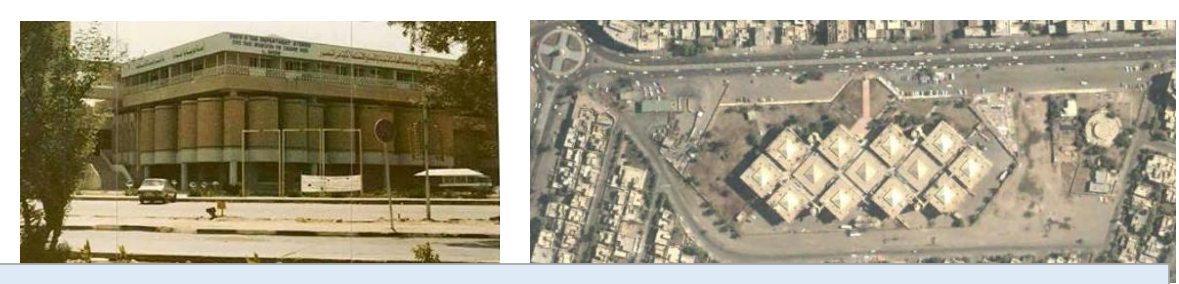

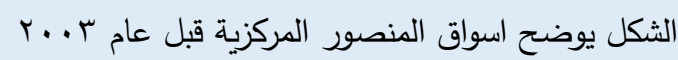

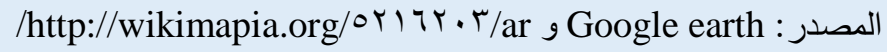



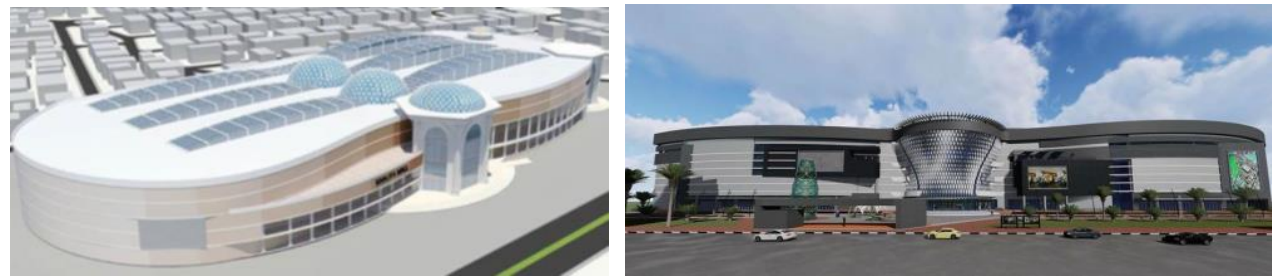

الثكل يوضح مول خليفة المزمع تتفيذه بموقع الاسواق المركزية

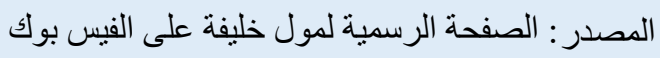
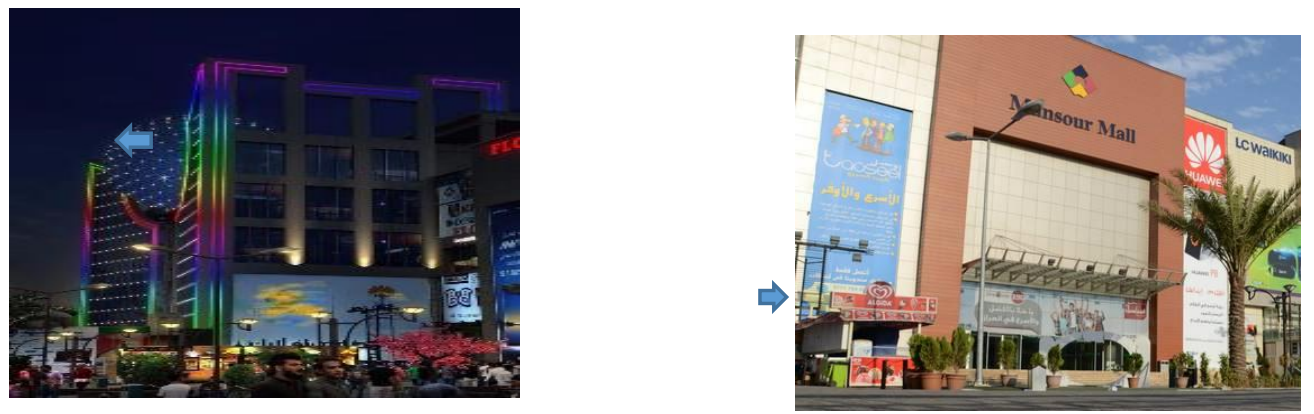

$$
\text { الثكل يوضح مول التاون سنتر }
$$

ץ- المباني التجارية: تتتشر على جانبي الثارع العديد من المباني التجارية التي تتراوح ارتفاعاتها من r^ طوابق وفي الغالب الاستعمال للطابق الارضي تجاري اما الطوابق الاخرى فتختلف فيها الفعاليات منها تجاريه، مكتبية، سكنية وغيرها r- محطة تعبة وقود الرسالة

ع - المركز الثقافي للطفل العراقي (سينما الفانوس السحري) ه- العقد: يوجد عقدتين في شارع المنصور الرئيسي في بداية الثارع وفي نهايته وهي: 


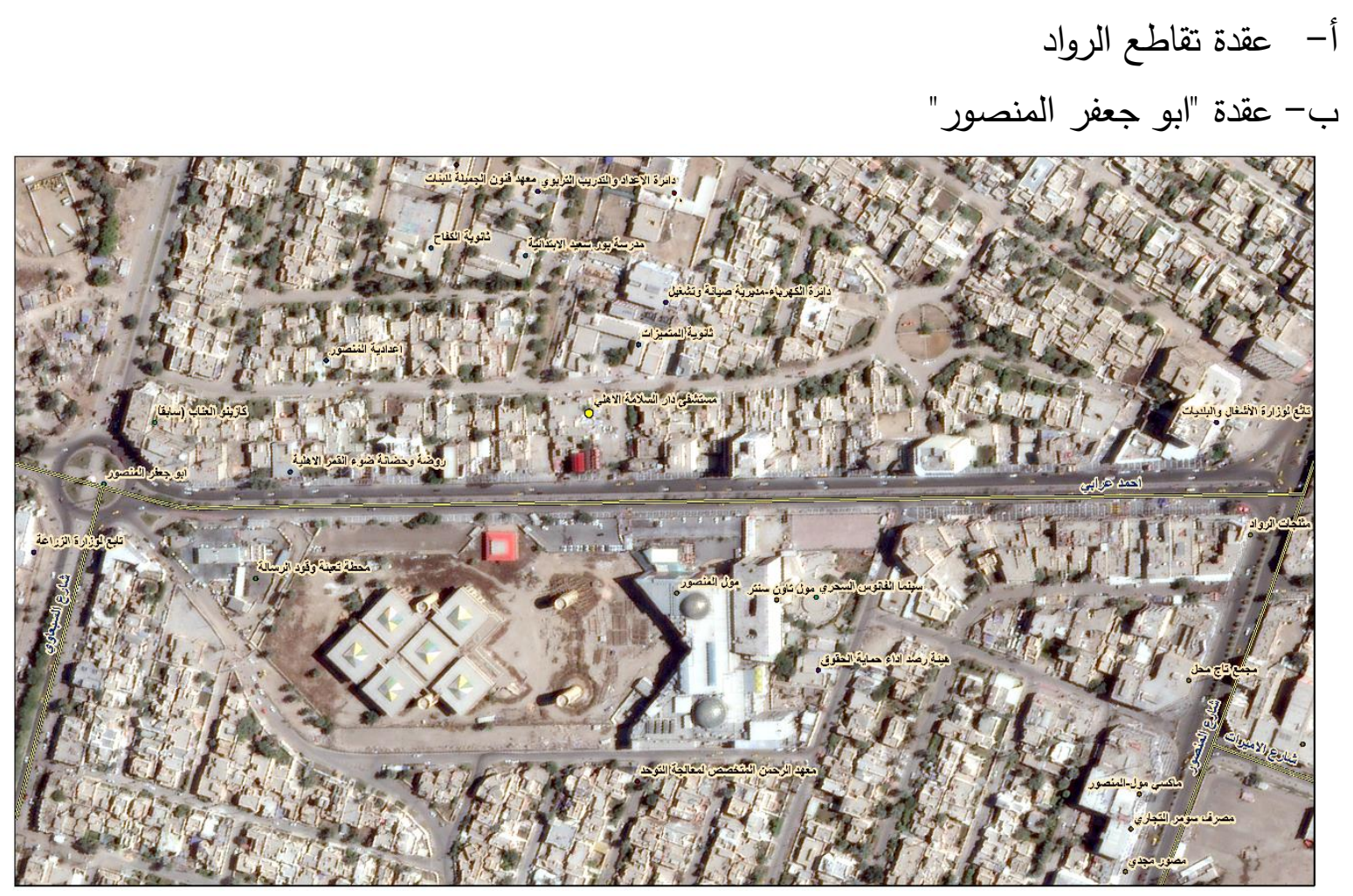

$$
\text { الثكل يوضح البنية الفضائية لثارع المنصور الرئيسي }
$$

الخصائص التركيبية للبنية الفضائية وتثمل

$$
\text { 1 - خصائص شمولية: }
$$

- - خاصية معدل العمق النسبي (Mean - depth): اظهر التحليل ان معدل العمق النسبي لجهة المراكز التجارية أكثر من الجهة المقابلة من الثارع 


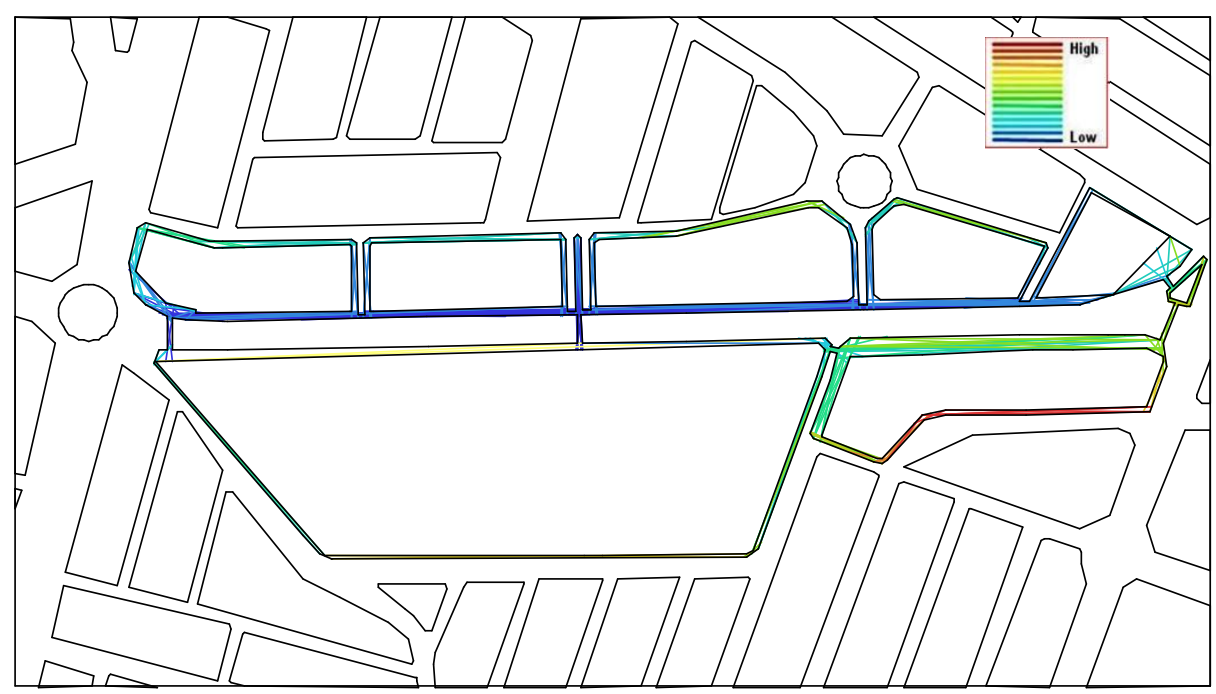

الثكل يوضح العمق النسبي للشارع// المصدر : الباحثة

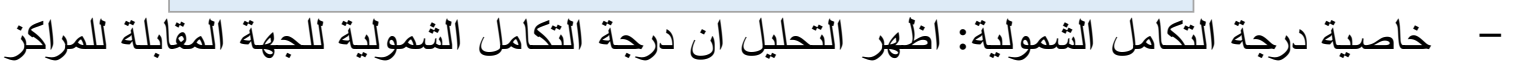
التجارية أكثر من جهة المراكز التجارية

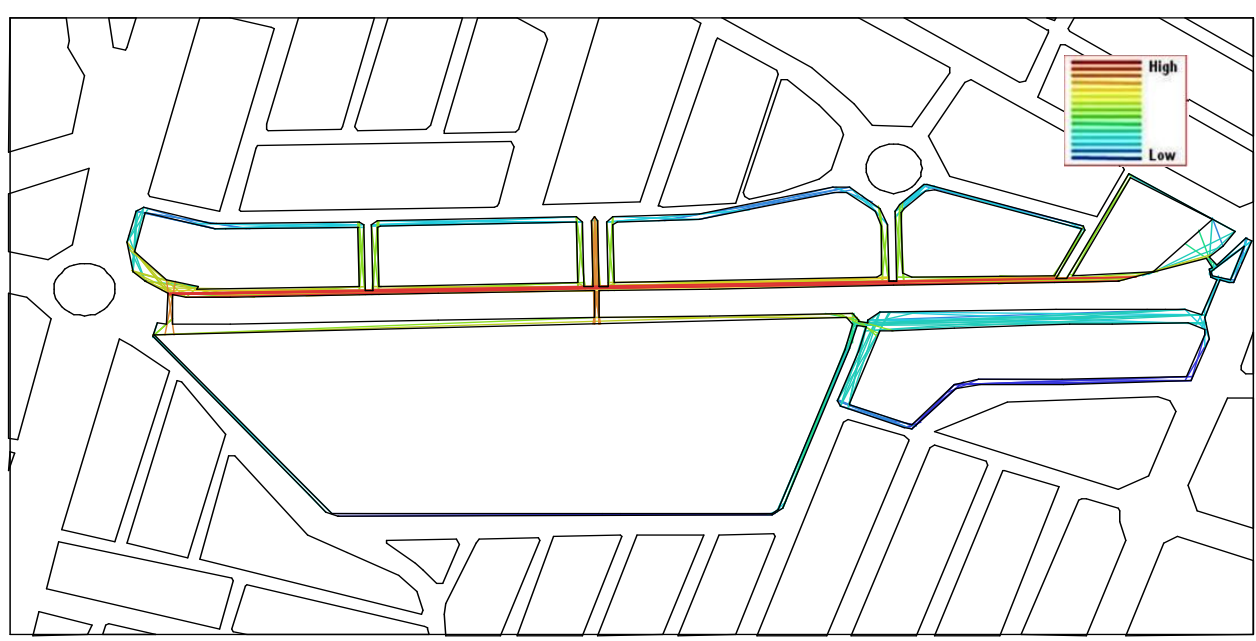

الثكل يوضح درجة التكامل الثمولية للشارع// المصدر : الباحثة 
- خاصية الخيار الثمولية (Global choice): اظهر التحليل ان درجة الخيار الثمولية لجهة المراكز التجارية أكثر من جهة المقابلة من الثارع

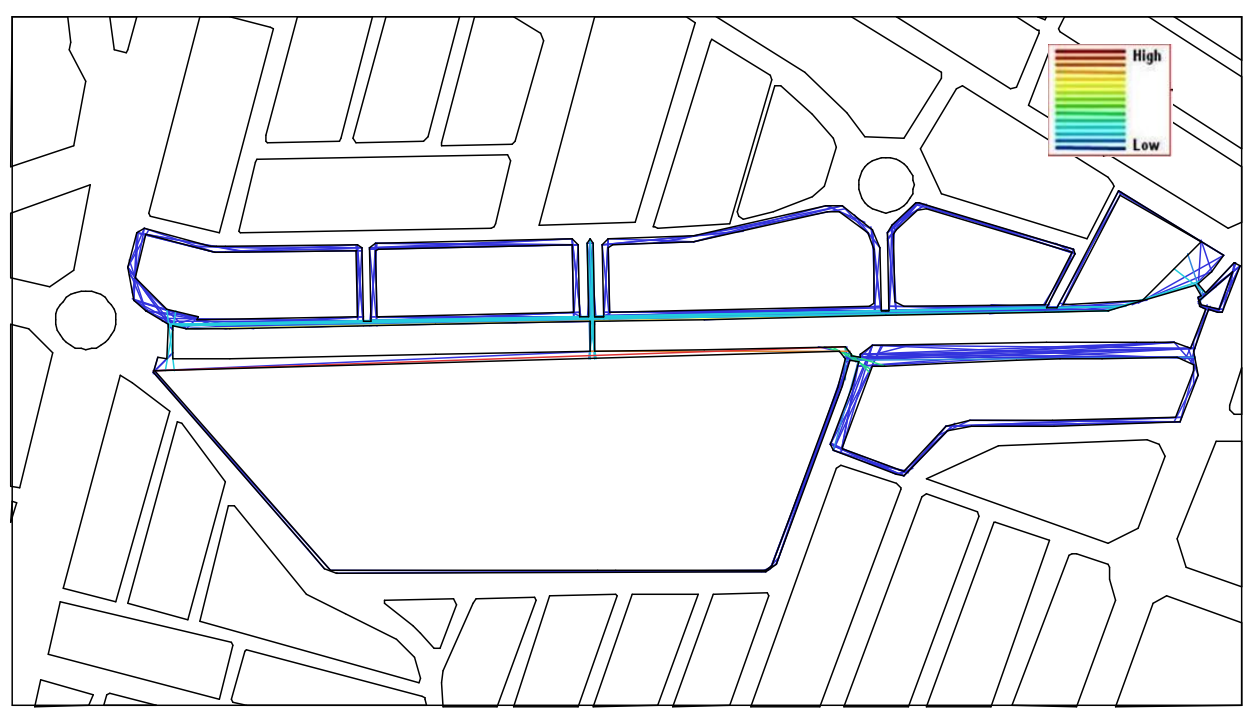

الشكل يوضح درجة الخيار الشمولية للشارع// المصدر : الباحثة

ז- الخصائص الموضعية: - خاصية الاتصالية (Connectivity): اظهر التحليل ان درجة الاتصالية الموضعية للجهة المقابلة للمراكز التجارية أكثر من جهة المراكز التجارية 


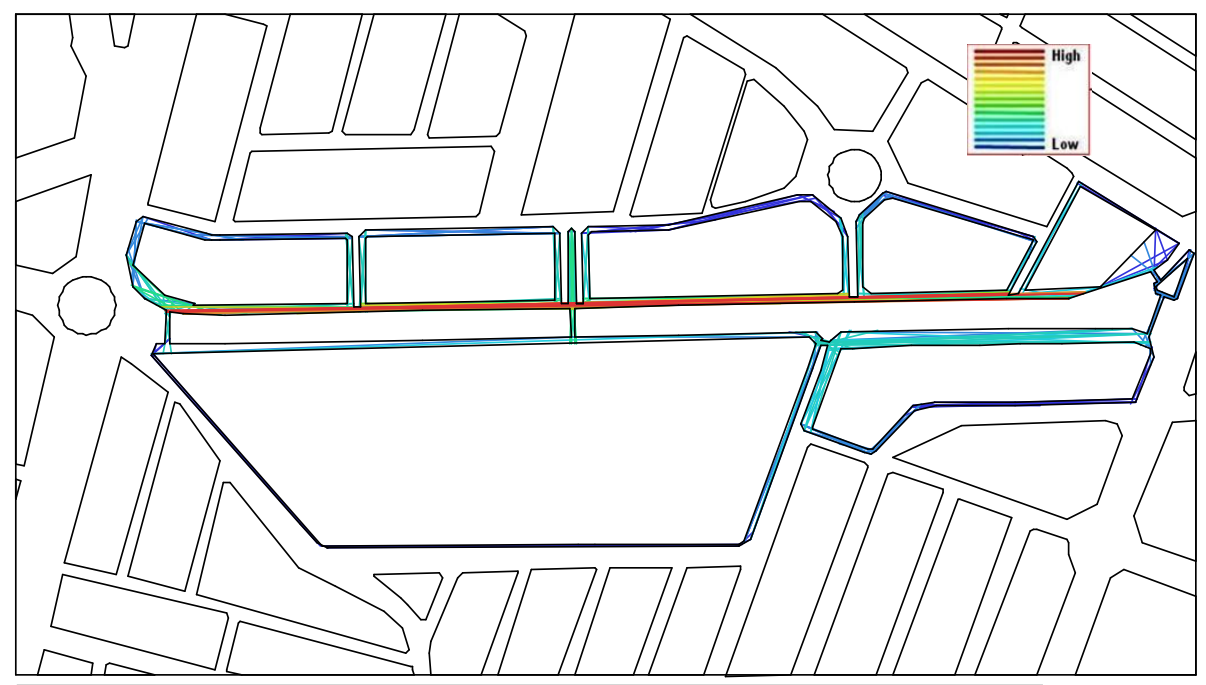

الثكل يوضح خاصية الاتصالية الموضعية للثارع// المصدر : الباحثة

- خاصية التكامل الموضعي (Local Integration): اظهر التحليل ان درجة التكامل الموضعي للجهة المقابلة للمراكز التجارية أكثر من جهة المراكز التجارية 


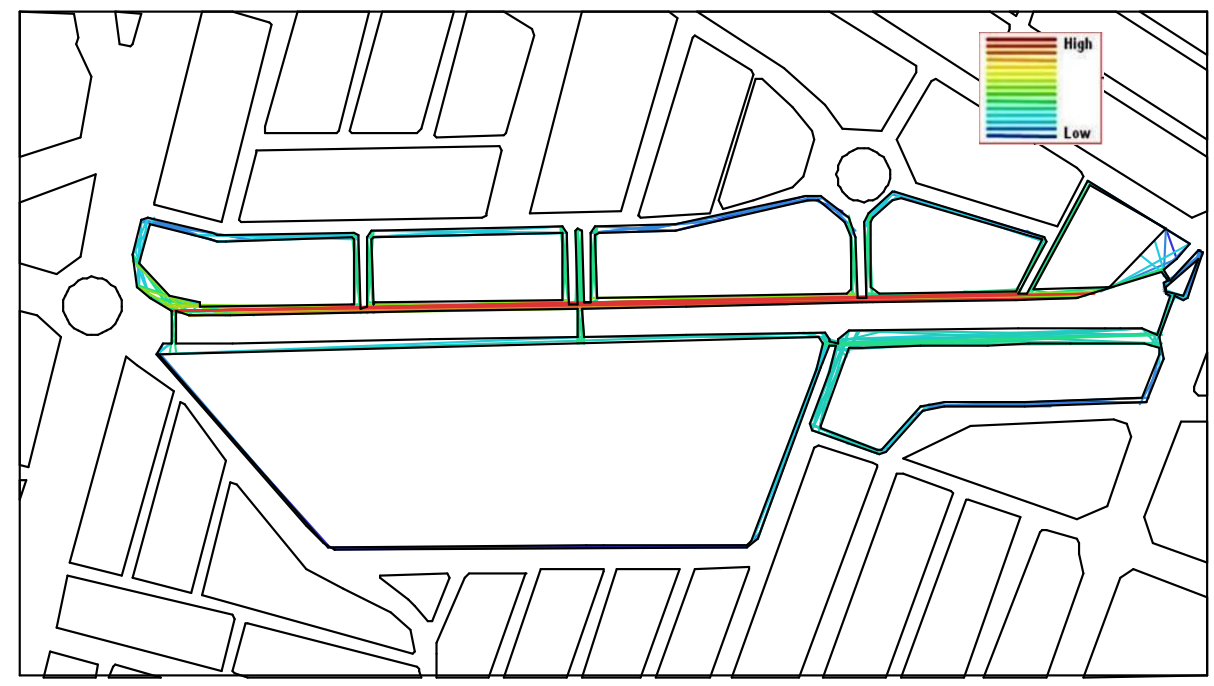

الشكل يوضح خاصية التكامل الموضعي للشارع// المصدر : الباحثة

r- الوضوحية التركيبية للشارع التجاري: الوضوحية في الثارع التجاري بنسبة V,· وبهذه النسبة يعتبر الثارع ذا درجة وضوحية جيدة

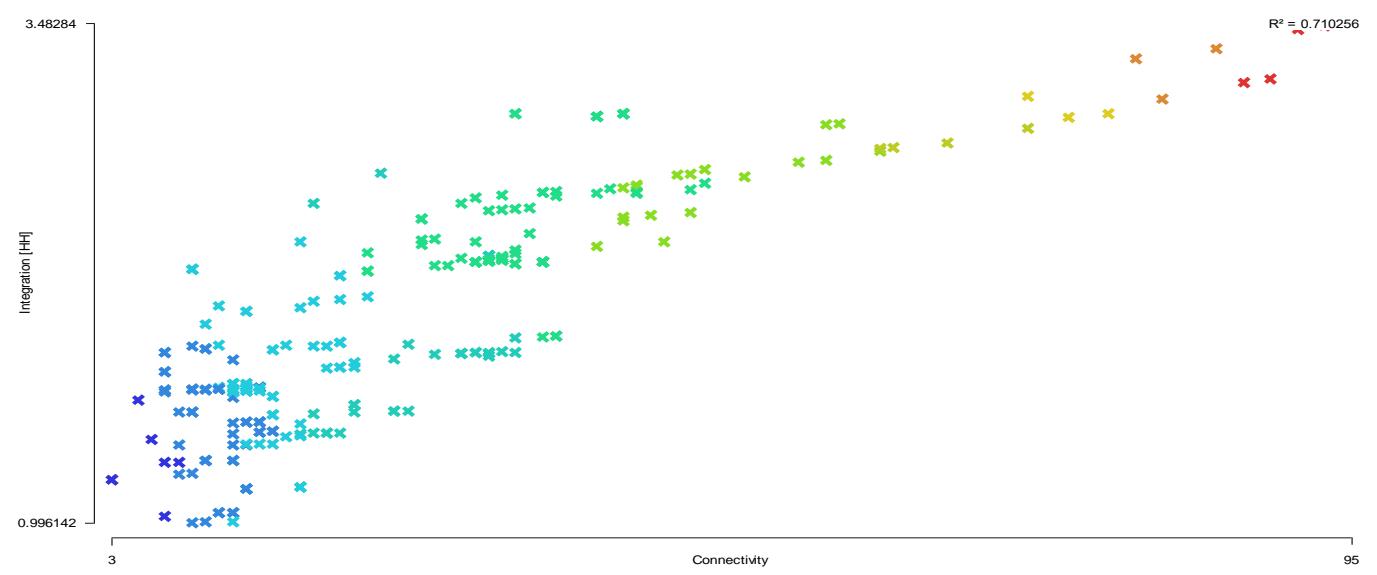

الشكل يوضح درجة الوضوحية للشارع// المصدر : الباحثة 
البنية السلوكية (الفعاليات): تتركز الفعاليات التجارية بالدرجة الاساسية والفعاليات الثقافية والتواصل الاجتماعي ايضا في مناطق المجمعات التجارية حيث يعتبر مكان لقاء للسكان أكثر من الجهة المقابلة للشارع. السلوك الحركي الفضائي: يتضح ان السلوك الحركي وفق البرنامج يتركز في جهة المراكز التجارية أكثر من الجهة المقابلة لها في الثارع ومن خلال المراقبة والتفحص ايضا يتضح تركز الحركة في جانب المراكز التجارية أكثر من الجانب المقابل

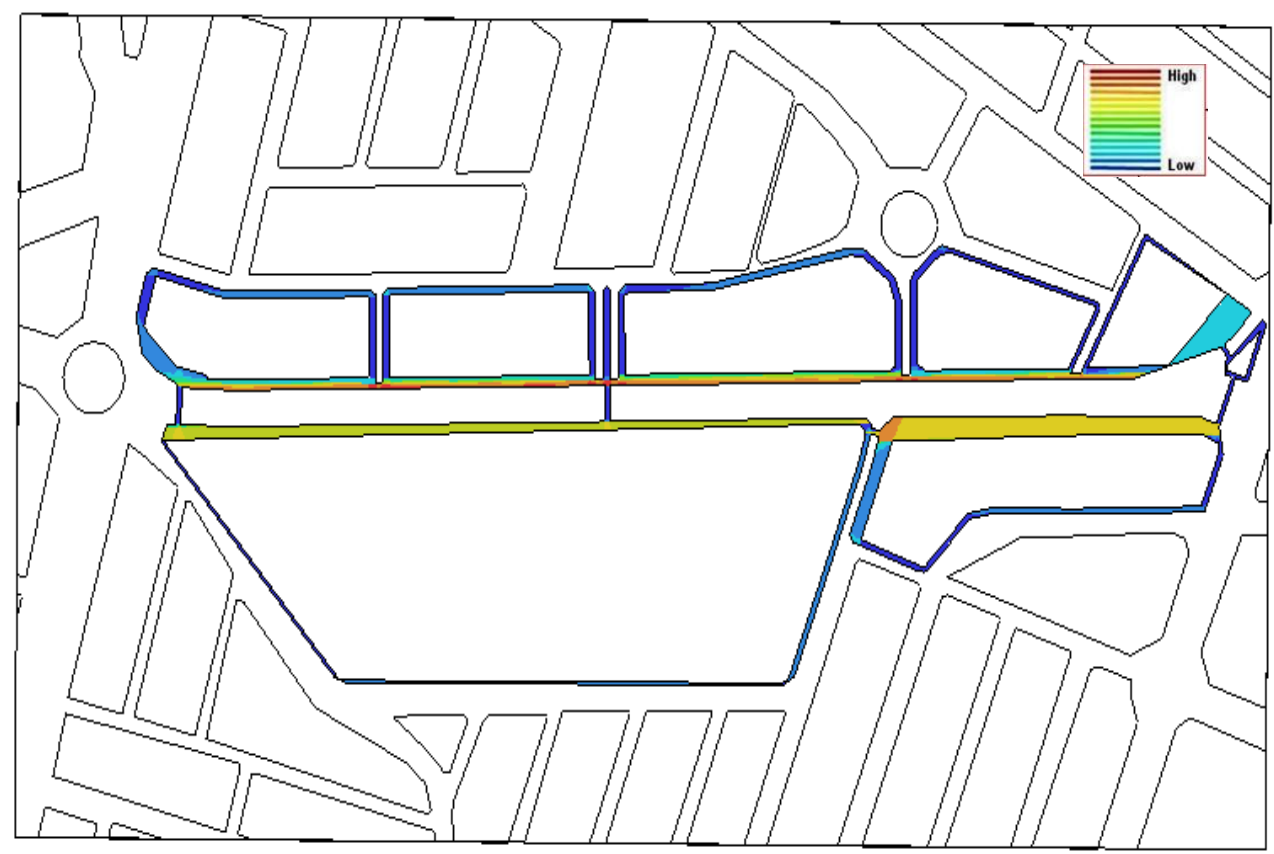

الثكل يوضح خاصية السلوك الحركي للشارع// الدصدر : الباحثة 
ا- ان الجهة المقابلة للمراكز التجارية تكون ذات درجة عالية في معدل درجة التكامل الثمولية والاتصالية الموضعية والتكامل الموضعي وهذا يدل على تماسك البنية الحضرية في هذه الجهة أكثر من الجهة الاخرى ولا يوجد انقطاع او تفكك في البنية اما الجهة المقابلة فيوجد انقطاع في بنيتها في منطقة الثارع الفرعي الذي لهي يفصل موقع المراكز التجارية عن الابنية التجارية في بداية الثارع بالقرب من تقاطع الرواد. ץ- جهة المراكز التجارية تتمتع بدرجة عالية من معدل العمق النسبي وخاصية الخيار الثمولية وهذا يدل على انها المسارات الاكثر استخداما من قبل المرتادين من الجهة المقابلة.

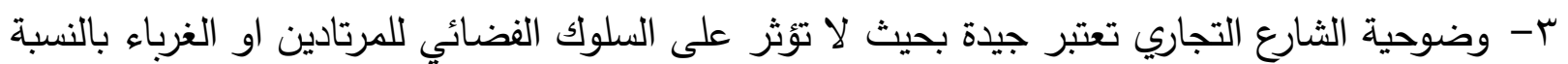
للشارع. ع - السلوك الفضائي للمتلقي يتركز في جهة المراكز التجارية التي كانت نسبة السلوك الحركي التركيبي فيها عالية وكذلك نسبة الخيار الموضعي التي تكون خصائص مميزة للفضاء لتحفز إدراك المتلقي فيه وبالتالي سلوكه الفضائي. المراجع: (الماجه 1- البياتي، طلال عباس ابراهيم، "التلوث البصري في الثوارع التجارية"، رسالة ماجستير مقدمة الى مركز التخطيط الحضري

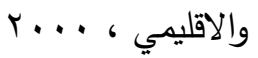

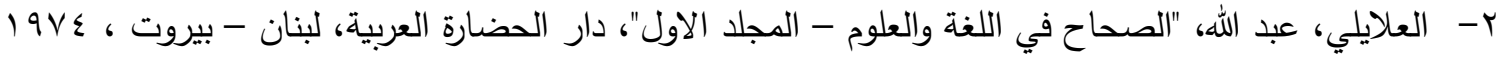
ץ- العلوان، هدى عبد الصاحب، "وضوحية البيئة المعمارية"، اطروحة دكتوراه مقدمة الى قسم الهندسة المعمارية، جامعة العالية

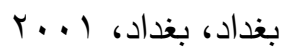
ع - زيدان، دينا اميل، "الفضاءات السالبة في العمارة / تأثير خصائص التنظيم الفضائي على اشغال الفضاءات المفتوحة" ،

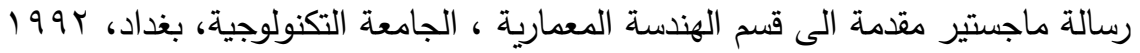
0- كلارك، سايمون، ترجمة سعيد العليمي، "اسس البنيوية نقد ليفي شتراوس والحركة البنيوية" ، دار بدائل ، الجيزة - مصر ، 
ч- Alexander, Christopher, " Anew Theory of Urban Design ", Oxford University Press; N.Y., USA, 19AV

V- Canter, David V. , "The psychology of place", the architectural press, London, I $9 \vee \vee$

^- Hillier, Bill \& Hanson, "The Social logic of Space" , Cambridge University Press, Cambridge, $191 \varepsilon$

9- Hillier, Bill, "Space is the machine", Cambridge university press, Cambridge, 1997

1.-Hillier, Bill, "The main ideas behind the space syntax method of spatial analysis and the historical pattern of architectural and planning" , United Kingdom, 19^^

11-Turner, Alasdair and Alan Penn, "Space Syntax Based Agent Simulation", UK, $. r . .1$

i $Y$-Rapoport, Amos, "Human aspect of urban form" , Towards Aman -environment approach to urban form and design, pergamon press, Oxford, U.K, $19 \mathrm{~V}$ 
